\title{
Asymptotic Analysis of the Curved-Pipe Flow with a Pressure-Dependent Viscosity Satisfying Barus Law
}

\author{
Igor Pažanin \\ Department of Mathematics, Faculty of Science, University of Zagreb, Bijenička 30, 10000 Zagreb, Croatia \\ Correspondence should be addressed to Igor Pažanin; pazanin@math.hr
}

Received 24 November 2014; Accepted 20 March 2015

Academic Editor: Ana Carpio

Copyright (C) 2015 Igor Pažanin. This is an open access article distributed under the Creative Commons Attribution License, which permits unrestricted use, distribution, and reproduction in any medium, provided the original work is properly cited.

\begin{abstract}
Curved-pipe flows have been the subject of many theoretical investigations due to their importance in various applications. The goal of this paper is to study the flow of incompressible fluid with a pressure-dependent viscosity through a curved pipe with an arbitrary central curve and constant circular cross section. The viscosity-pressure dependence is described by the well-known Barus law extensively used by the engineers. We introduce the small parameter $\varepsilon$ (representing the ratio of the pipe's thickness and its length) into the problem and perform asymptotic analysis with respect to $\varepsilon$. The main idea is to rewrite the governing problem using the appropriate transformation and then to compute the asymptotic solution using curvilinear coordinates and two-scale asymptotic expansion. Applying the inverse transformation, we derive the asymptotic approximation of the flow clearly showing the influence of pipe's distortion and viscosity-pressure dependence on the effective flow.
\end{abstract}

\section{Introduction}

Curved-pipe flows have gained much attention over past years due to their importance in numerous industrial and engineering applications. Air conditioners, refrigeration systems, central heating radiators, and chemical reactors are only few examples of devices where we can find different types of curved pipes. From the theoretical point of view, curved-pipe flows are interesting due to the appearance of secondary flows caused by the effects of the pipe's distortion. Therefore, when analyzing such problems, the main attempt is to detect the effects of the pipe's geometry on the velocity and pressure distribution through the pipe. The engineering approach to the curved-pipe flows is often based on the Poiseuille formula providing an exact solution only in case of stationary, laminar Newtonian flow through a straight pipe with constant cross section. However, if the pipe is curved, such formula only gives an approximation of the solution with low order of accuracy. In view of that, the Poiseuille flow has to be corrected by the lower-order term which contains the effects of pipe's curvedness leading to a more accurate approximation.

In his celebrated work, Stokes [1] suggested that the viscosity of the fluid can depend on the pressure. Since then, numerous researchers confirmed that, especially at high values of pressure, the variations of the viscosity with pressure should be taken into account while the flow is still incompressible. For that reason, the problem of incompressible fluid flow with a pressure-dependent viscosity is very attractive and has been extensively studied in recent years, mostly in the engineering literature (see, e.g., [2-6]). There exist several ways to describe the viscosity-pressure relation. Among all, the most famous one is, without any doubt, the Barus law [7]:

$$
\mu(p)=\mu_{0} e^{\beta p} .
$$

Here $\mu_{0}$ stands for the viscosity at atmospheric pressure while $\beta>0$ is the pressure-viscosity coefficient. Barus formula has been extensively used throughout the engineering literature.

Motivated by the above discussion, the aim of this paper is to study the incompressible fluid with a pressure-dependent viscosity obeying Barus law and flowing through a curved pipe with constant circular cross section. Introducing the viscosity-pressure dependence (1) into the Navier-Stokes system completely changes the nature of the system making it very challenging from the mathematical point of view. It brings the additional nonlinearity to the system and the flow becomes non-Newtonian. The main difficulty lies in the 
fact that we cannot treat pressure as we did in the classical, Newtonian case so we need to change our approach. In view of that, our strategy consists of the following three steps:

(1) rewriting the governing system by replacing the original pressure with a new, transformed pressure;

(2) finding the solution of the transformed system satisfied by the velocity and a new pressure;

(3) reconstructing the effective pressure by applying the inverse transformation.

Naturally, it is not reasonable to expect that we will succeed to find the exact solution of the governing 3D boundaryvalue problem. Therefore, inspired by the applications, we introduce the small parameter $\varepsilon$ into the system (denoting the ratio between pipe's thickness and its length) and consider the flow in a pipe which is either very thin or very long. By doing that, we are in position to perform the asymptotic analysis as $\varepsilon \rightarrow 0$ and to build the asymptotic approximation of the flow with high order of accuracy.

Along with the viscosity-pressure dependence, our aim is to treat as general domain as possible. Thus, we assume that the pipe's central curve, denoted by $\gamma$, is an arbitrary smooth curve given by its natural parametrization. The only constraint we impose on $\gamma$ is that it is a generic curve. As a consequence, we can use local Frenet's basis attached to $\gamma$ and use the curvilinear coordinates to formally define our domain. An efficient technique for handling curved geometries has been proposed in some of our previous works (see, e.g., [8-10]) and we employ it here to construct the asymptotic solution of the transformed system (Step 2). It enables us to explicitly compute the terms from the two-scale asymptotic expansion and to detect the effects we seek for. Indeed, after applying the inverse transformation, we obtain the asymptotic approximation for the velocity and pressure explicitly acknowledging the effects of pressure-dependent viscosity and the pipe's curvedness. By taking those effects into account, we believe that the obtained result is very relevant with regard to numerical simulations and could improve the known engineering practice. We should also mention that the presented approach can be generalized to a case of a general viscosity-pressure relation, as commented in the concluding section.

We conclude the introduction by providing more bibliographic remarks on the subject. Curved-pipe flow in case of constant viscosity ( $\mu=$ const.) has been extensively investigated for various liquids and regime of flows; see, for example, [11-15]. In case of pressure-dependent viscosity, to our knowledge there are no analytical results on the curvedpipe flows. Analytical solutions have been reported only in some simplified situations like unidirectional and planeparallel flows and under the assumption of the linear law $\mu(p)=\beta p$ or $\mu(p)=\mu_{0}(1+\beta p)$. We refer the reader to [16-20]. However, a year ago, the straight-pipe flow has been successfully addressed by Marušić-Paloka and Pažanin [21] in the case of exponential law (1). The flow through a specific helical pipe frequently appearing in the applications has been analyzed this year in [22]. The goal of the present paper is to extend the analysis presented in $[21,22]$ to a general framework, that is, the case of general curved pipe.

\section{Position of the Problem}

2.1. The Pipe's Geometry. In this section we formally describe the complex pipe's geometry. As emphasized in Introduction, we want to address the case of a general curved pipe with circular cross section. In view of that, we introduce a generic curve in $\mathbf{R}^{3}$, denoted by $\gamma$, which serves to define the central curve of the pipe. We suppose that $\gamma$ is parameterized by its arc length $x_{1} \in[0, \ell]$ and denote by $\pi \in C^{3}\left([0, \ell] ; \mathbf{R}^{3}\right)$ its natural parametrization. We also assume that $\pi^{\prime}\left(x_{1}\right) \neq 0$, for every $x_{1} \in[0, \ell]$. Since $\gamma$ is taken to be generic, it holds $\left|\pi^{\prime \prime}\left(x_{1}\right)\right|>0$, for every $x_{1} \in[0, \ell]$. Denoting by $\kappa\left(x_{1}\right)=$ $\left|\pi^{\prime \prime}\left(x_{1}\right)\right|$ the flexion of the curve $\gamma$, we introduce Frenet's basis in a standard way:

$$
\begin{gathered}
\mathbf{t}=\pi^{\prime} \quad(\text { the tangent }), \\
\mathbf{n}=\frac{1}{\kappa} \mathbf{t}^{\prime} \quad(\text { the normal }), \\
\mathbf{b}=\mathbf{t} \times \mathbf{n} \quad \text { (the binormal). }
\end{gathered}
$$

The normal $\mathbf{n}$ is extended by continuity in points where curvature is zero. We also denote by $\tau\left(x_{1}\right)=-\left|\mathbf{b}^{\prime}\left(x_{1}\right)\right|$ the torsion of $\gamma$. One of the main goals of this study is to detect the influence of geometric parameters $\kappa$ and $\tau$ on the effective flow.

Next, we introduce the small parameter $\varepsilon(0<\varepsilon \ll 1)$ into the problem and first define an undeformed pipe

$$
T_{\varepsilon}=\left\{x=\left(x_{1}, x_{2}, x_{3}\right) \in \mathbf{R}^{3}: x_{1} \in(0, \ell), \tilde{x}=\left(x_{2}, x_{3}\right) \in \varepsilon B\right\},
$$

where $B=B(0,1)=\left\{y \in \mathbf{R}^{2}:|y|<1\right\}$ is the unit circle. Now we have to choose the appropriate parametrization to define our curved pipe. The best way to do this is to introduce the mapping $\Phi_{\varepsilon}^{\alpha}: T_{\varepsilon} \rightarrow \mathbf{R}^{3}$ as follows:

$$
\Phi_{\varepsilon}^{\alpha}(x)=\pi\left(x_{1}\right)+x_{2} \mathbf{n}_{\alpha}\left(x_{1}\right)+x_{3} \mathbf{b}_{\alpha}\left(x_{1}\right) .
$$

Here

$$
\begin{aligned}
& \mathbf{n}_{\alpha}\left(x_{1}\right)=\cos \alpha\left(x_{1}\right) \mathbf{n}\left(x_{1}\right)+\sin \alpha\left(x_{1}\right) \mathbf{b}\left(x_{1}\right) \\
& \mathbf{b}_{\alpha}\left(x_{1}\right)=-\sin \alpha\left(x_{1}\right) \mathbf{n}\left(x_{1}\right)+\cos \alpha\left(x_{1}\right) \mathbf{b}\left(x_{1}\right)
\end{aligned}
$$

stand for the rotated unit vectors with respect to standard Frenet's normal and binormal (see Figure 1), where the rotation is given by

$$
\alpha\left(x_{1}\right)=-\int_{x_{0}}^{x_{1}} \tau(\xi) d \xi+\alpha_{0}, \quad\left(x_{0}, \alpha_{0} \text { arbitrary constants }\right) .
$$

Observe that by putting $\alpha \equiv 0$ we get classical Frenet's system which is most usually employed in describing the curved geometries. However, since we analyze the problem 


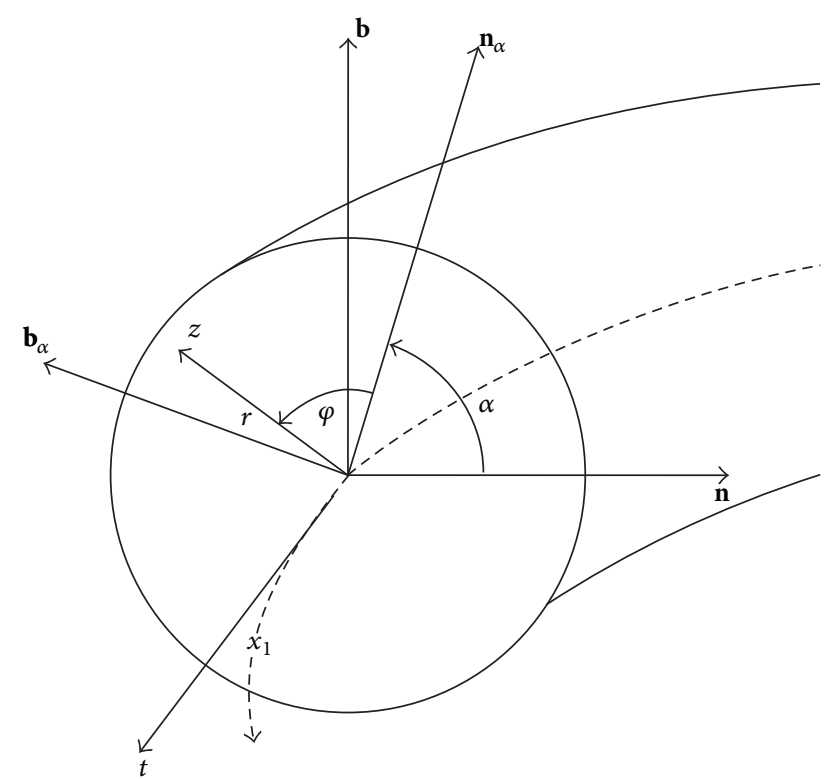

FIGURE 1: The reference system.

in the pipe with circular cross section (the most common one in the engineering applications), it is plausible to use this particular reference system in which the domain's cross section possesses no rotation with respect to the tangent vector $\mathbf{t}$. Let us mention that it was originally introduced by Germano in the 80s for treating the classical Newtonian flow through a helically coiled pipe (see $[23,24])$.

We are now in position to define our three-dimensional domain representing thin (or long) curved pipe with an arbitrary central curve $\gamma$ and circular cross section. It is given by

$$
\Omega_{\varepsilon}^{\alpha}=\Phi_{\varepsilon}^{\alpha}\left(T_{\varepsilon}\right)
$$

Finally, we denote by $\Sigma_{\varepsilon}^{i}=\Phi_{\varepsilon}^{\alpha}(\{i\} \times \varepsilon B), i=0, \ell$, the pipe's ends and by $\Gamma_{\varepsilon}^{\alpha}=\Phi_{\varepsilon}^{\alpha}((0, \ell) \times \varepsilon \partial B)$ the lateral boundary.

Remark 1. From the strictly mathematical point of view, we have to ensure the local injectivity of the parametrization $\Phi_{\varepsilon}^{\alpha}$. It can be accomplished by assuming that $\varepsilon$ is sufficiently small; namely, $\varepsilon\|\kappa\|_{L^{\infty}(0, \ell)}<1 / 2$ (see (29)).

2.2. The Governing System. We suppose that the pipe $\Omega_{\varepsilon}^{\alpha}$ is filled with incompressible fluid with a viscosity depending on pressure. For such fluids, the stress tensor can be taken as

$$
\mathbf{T}=-p \mathbf{I}+2 \mu(p) \mathbf{D}(\mathbf{u})
$$

where $\mathbf{D}(\mathbf{u})=(1 / 2)\left[\nabla \mathbf{u}+(\nabla \mathbf{u})^{T}\right]$ stands for the symmetric part of the velocity gradient. We can always assume that the Reynolds number is not too large and neglect the inertial term in the original Navier-Stokes system. In view of that, the flow in $\Omega_{\varepsilon}^{\alpha}$ is going to be governed by the following system for the unknown velocity $\mathbf{u}_{\varepsilon}$ and pressure $p_{\varepsilon}$ :

$$
\begin{gathered}
-\operatorname{div}\left[2 \mu\left(p_{\varepsilon}\right) \mathbf{D}\left(\mathbf{u}_{\varepsilon}\right)\right]+\nabla p_{\varepsilon}=0 \quad \text { in } \Omega_{\varepsilon}^{\alpha}, \\
\operatorname{div} \mathbf{u}_{\varepsilon}=0 \quad \text { in } \Omega_{\varepsilon}^{\alpha} .
\end{gathered}
$$

As mentioned before, we use the well-known Barus law to describe the viscosity-pressure dependence:

$$
\mu(p)=\mu_{0} e^{\beta p}, \quad \mu_{0}, \beta=\text { const. }>0 .
$$

The above system should be completed by the appropriate boundary conditions. Since our aim is to study a real-life situation, we assume that the flow is governed by the prescribed pressure drop between pipe's ends. On the lateral part of the boundary we prescribe the classical no-slip boundary condition for the velocity:

$$
\begin{gathered}
\mathbf{u}_{\varepsilon}=0 \quad \text { on } \Gamma_{\varepsilon}^{\alpha}, \\
\mathbf{u}_{\varepsilon} \times \mathbf{t}=0, \quad p^{\varepsilon}=p_{i} \quad \text { on } \Sigma_{\varepsilon}^{i}, i=0, \ell .
\end{gathered}
$$

Here $p_{0}$ and $p_{\ell}$ are the given constant pressures $\left(p_{0}>p_{\ell}\right)$. The well-posedness of the above problem has been recently established by Marušić-Paloka [25]. Our goal is to find the asymptotic behavior of the flow, as the thickness $\varepsilon \rightarrow 0$.

\section{Asymptotic Modeling}

3.1. Transformed System. Substituting the Barus law into the momentum equation (9) leads to

$$
\begin{aligned}
0 & =-\operatorname{div}\left[2 \mu_{0} e^{\beta p_{\varepsilon}} \mathbf{D}\left(\mathbf{u}_{\varepsilon}\right)\right]+\nabla p_{\varepsilon} \\
& =-\mu_{0} e^{\beta p_{\varepsilon}} \Delta \mathbf{u}_{\varepsilon}-2 \mu_{0} \beta e^{\beta p_{\varepsilon}} \mathbf{D}\left(\mathbf{u}_{\varepsilon}\right) \nabla p_{\varepsilon}+\nabla p_{\varepsilon} .
\end{aligned}
$$

We divide it by $\mu_{0} e^{\beta p_{\varepsilon}}$ to obtain

$$
-\Delta \mathbf{u}_{\varepsilon}+\frac{1}{\mu_{0}} e^{-\beta p_{\varepsilon}} \nabla p_{\varepsilon}=2 \beta \mathbf{D}\left(\mathbf{u}_{\varepsilon}\right) \nabla p_{\varepsilon} .
$$

The form of the above equation suggests introducing a new function, denoted by $q_{\varepsilon}$, such that

$$
\frac{1}{\mu_{0}} e^{-\beta p_{\varepsilon}} \nabla p_{\varepsilon}=\nabla q_{\varepsilon}
$$

Here and in the sequel we will call it the transformed pressure. It is obvious that (15) will be satisfied if we take

$$
q_{\varepsilon}=\frac{1}{\beta \mu_{0}}\left(e^{-\beta q_{0}}-e^{-\beta p_{\varepsilon}}\right), \quad q_{0} \in \mathbf{R} \text { arbitrary. }
$$

By a simple calculation we deduce

$$
\nabla p_{\varepsilon}=\mu_{0} e^{\beta p_{\varepsilon}} \nabla q_{\varepsilon}=\frac{\mu_{0}}{e^{-\beta q_{0}}-\beta \mu_{0} q_{\varepsilon}} \nabla q_{\varepsilon} .
$$

Consequently, (14) becomes

$$
-\Delta \mathbf{u}_{\varepsilon}+\nabla q_{\varepsilon}=\frac{2 \beta \mu_{0}}{e^{-\alpha q_{0}}-\beta \mu_{0} q_{\varepsilon}} \mathbf{D}\left(\mathbf{u}_{\varepsilon}\right) \nabla q_{\varepsilon} .
$$


The above procedure yields the following system satisfied by the velocity $\mathbf{u}^{\varepsilon}$ and the transformed pressure $q^{\varepsilon}$ (we will refer to it as the transformed system):

$$
\begin{gathered}
-\Delta \mathbf{u}_{\varepsilon}+\nabla q_{\varepsilon}=\frac{2 \beta \mu_{0}}{e^{-\beta q_{0}-\beta \mu_{0} q^{\varepsilon}}} \mathbf{D}\left(\mathbf{u}_{\varepsilon}\right) \nabla q_{\varepsilon} \quad \text { in } \Omega_{\varepsilon}^{\alpha}, \\
\operatorname{div} \mathbf{u}_{\varepsilon}=0 \quad \text { in } \Omega_{\varepsilon}^{\alpha}, \\
\mathbf{u}_{\varepsilon}=0 \quad \text { on } \Gamma_{\varepsilon}^{\alpha}, \\
\mathbf{u}_{\varepsilon} \times \mathbf{t}=0, \quad q_{\varepsilon}=\frac{1}{\beta \mu_{0}}\left(e^{-\beta q_{0}}-e^{-\beta p_{i}}\right) \quad \text { on } \Sigma_{\varepsilon}^{i}, i=0, \ell .
\end{gathered}
$$

The obtained transformed system is in the form of a nonlinear Stokes-like system (with nonlinearity appearing on the right-hand side in (19)) and it will be the subject of our investigation in the sequel. More precisely, after writing the transformed system in curvilinear coordinates $\left(x_{i}\right)$, we will construct its asymptotic solution via two-scale asymptotic technique. The liberty in choice of parameter $q_{0}$ (see (16)) will enable us to control the nonlinearity in a way that it does not contribute to the macroscopic model. Indeed, we can always choose $q_{0}$ small enough such that

$$
\lim _{q_{0} \rightarrow-\infty} \frac{\beta \mu_{0}}{e^{-\beta q_{0}}-\beta \mu_{0} q^{\varepsilon}}=0 .
$$

It is essential to be aware that, throughout the whole process, $q_{0}$ plays the role of an auxiliary parameter. It means that, by choosing $q_{0}$ such that (23) holds, we do not impose any additional constraints since it turns out that the effective pressure will not depend on the parameter $q_{0}$ at all (see Section 3.4.).

\subsection{Transformed System in Curvilinear Coordinates}

3.2.1. Tools from Differential Geometry. In order to write the transformed equations (19)-(20) in the curvilinear coordinates $\left(x_{i}\right)$, we need to introduce some advanced notions from differential geometry. We begin by introducing the covariant basis defined as

$$
\mathbf{a}_{i}(x)=\frac{\partial \Phi_{\varepsilon}^{\alpha}}{\partial x_{i}}(x), \quad i=1,2,3 .
$$

Using the fact that $\alpha^{\prime}=-\tau$ together with

$$
\mathbf{t}^{\prime}=\kappa \mathbf{n}, \quad \mathbf{n}^{\prime}=-\kappa \mathbf{t}+\tau \mathbf{b}, \quad \mathbf{b}^{\prime}=-\tau \mathbf{n},
$$

it is straightforward to obtain

$$
\begin{aligned}
& \mathbf{a}_{1}=\left(1-\kappa\left(e_{\alpha} \cdot \tilde{x}\right)\right) \mathbf{t}, \\
& \mathbf{a}_{2}=\cos \alpha \mathbf{n}+\sin \alpha \mathbf{b}, \\
& \mathbf{a}_{3}=-\sin \alpha \mathbf{n}+\cos \alpha \mathbf{b} .
\end{aligned}
$$

To make the complex notation more compact, here and in the sequel we introduce

$$
e_{\alpha}=(\cos \alpha,-\sin \alpha), \quad e_{\alpha}^{\perp}=(\sin \alpha, \cos \alpha)
$$

and denote by $\cdot$ the standard scalar product in $\mathbf{R}^{2}$. We also denote $\mathbf{B}=\left[\begin{array}{lll}\mathbf{t} & \mathbf{n} & \mathbf{b}\end{array}\right]$ implying

$$
\nabla \boldsymbol{\Phi}_{\varepsilon}^{\alpha}=\mathbf{B}\left[\begin{array}{ccc}
1-\kappa\left(e_{\alpha} \cdot \tilde{x}\right) & 0 & 0 \\
0 & \cos \alpha & -\sin \alpha \\
0 & \sin \alpha & \cos \alpha
\end{array}\right]
$$

Observe that

$$
\operatorname{det} \nabla \Phi_{\varepsilon}^{\alpha}=1-\kappa\left(e_{\alpha} \cdot \tilde{x}\right) .
$$

We employed this fact to assure the local injectivity of the parametrization $\boldsymbol{\Phi}_{\varepsilon}^{\alpha}$ (see Remark 1).

The covariant basis is complemented with the contravariant basis given by the relation

$$
\mathbf{a}^{i} \cdot \mathbf{a}_{j}=\delta_{i j}, \quad i, j=1,2,3 .
$$

In our case it reads

$$
\begin{gathered}
\mathbf{a}^{1}=\frac{1}{1-\kappa\left(e_{\alpha} \cdot \tilde{x}\right)} \mathbf{t}, \\
\mathbf{a}^{2}=\cos \alpha \mathbf{n}+\sin \alpha \mathbf{b}, \\
\mathbf{a}^{3}=-\sin \alpha \mathbf{n}+\cos \alpha \mathbf{b} .
\end{gathered}
$$

Finally, Christoffel's symbols are defined as

$$
\Gamma_{j k}^{i}=\mathbf{a}^{i} \cdot \frac{\partial \mathbf{a}_{k}}{\partial x_{j}}, \quad i, j, k=1,2,3 .
$$

Its fundamental property is that they are symmetric in lower indices; that is, $\Gamma_{j k}^{i}=\Gamma_{k j}^{i}$. We leave the reader to confirm that nonzero Christoffel's symbols in our setting are given by

$$
\begin{gathered}
\Gamma_{11}^{1}=-\frac{\left(\kappa^{\prime}\left(e_{\alpha} \cdot \tilde{x}\right)+\kappa \tau\left(e_{\alpha}^{\perp} \cdot \tilde{x}\right)\right)}{1-\kappa\left(e_{\alpha} \cdot \tilde{x}\right)}, \\
\Gamma_{12}^{1}=\Gamma_{21}^{1}=-\frac{\kappa \cos \alpha}{1-\kappa\left(e_{\alpha} \cdot x_{*}\right)}, \\
\Gamma_{13}^{1}=\Gamma_{31}^{1}=\frac{\kappa \sin \alpha}{1-\kappa\left(e_{\alpha} \cdot \tilde{x}\right)}, \\
\Gamma_{11}^{2}=\kappa\left(1-\kappa\left(e_{\alpha} \cdot \tilde{x}\right)\right) \cos \alpha, \\
\Gamma_{11}^{3}=-\kappa\left(1-\kappa\left(e_{\alpha} \cdot \tilde{x}\right)\right) \sin \alpha .
\end{gathered}
$$

Now we are going to establish the asymptotic behavior of the above quantities needed in the sequel. Having in mind that $x_{2}, x_{3}=\mathcal{O}(\varepsilon)$, from (29), we first conclude that $\operatorname{det} \nabla \Phi_{\varepsilon}^{\alpha}=$ $\mathcal{O}(1)$. Next, we have

$$
\left(\nabla \Phi_{\varepsilon}^{\alpha}\right)^{-1}=\left[\begin{array}{ccc}
1+\varepsilon \kappa\left(e_{\alpha} \cdot y\right) & 0 & 0 \\
0 & \cos \alpha & \sin \alpha \\
0 & -\sin \alpha & \cos \alpha
\end{array}\right] \mathbf{B}^{T}+\mathcal{O}\left(\varepsilon^{2}\right)
$$


where $y=\left(y_{2}, y_{3}\right), y_{i}=x_{i} / \varepsilon, i=2,3$. Here we used the fact that the vectors of the contravariant basis are, in fact, the rows of $\left(\nabla \Phi_{\varepsilon}^{\alpha}\right)^{-1}$. Finally, for Christoffel's symbols we obtain

$$
\begin{gathered}
\Gamma_{11}^{1}=\mathcal{O}(\varepsilon) \\
\Gamma_{12}^{1}=\Gamma_{21}^{1}=-\kappa \cos \alpha+\mathcal{O}(\varepsilon), \\
\Gamma_{13}^{1}=\Gamma_{31}^{1}=\kappa \sin \alpha+\mathcal{O}(\varepsilon) \\
\Gamma_{11}^{2}=\kappa \cos \alpha+\mathcal{O}(\varepsilon) \\
\Gamma_{11}^{3}=-\kappa \sin \alpha+\mathcal{O}(\varepsilon) .
\end{gathered}
$$

3.2.2. The Equations in Curvilinear Coordinates. In [10], the reader can find detailed derivation of the following formulae for differential operators in curvilinear coordinates:

$$
\begin{gathered}
(\nabla s)^{T} \circ \Phi_{\varepsilon}^{\alpha}=\left(\nabla \Phi_{\varepsilon}^{\alpha}\right)^{-T}(\nabla S)^{T} \\
(\nabla \mathbf{v}) \circ \Phi_{\varepsilon}^{\alpha}=\left(\nabla \Phi_{\varepsilon}^{\alpha}\right)^{-T}\left(\left[\frac{\partial V^{k}}{\partial x_{l}}\right]_{k, l}-V^{j} \Gamma^{j}\right)\left(\nabla \Phi_{\varepsilon}^{\alpha}\right)^{-1} \\
\Gamma^{i}=\left[\Gamma_{j k}^{i}\right]_{j, k}, \\
(\Delta \mathbf{v}) \circ \Phi_{\varepsilon}^{\alpha} \\
=\left(\nabla \Phi_{\varepsilon}^{\alpha}\right)^{-T}\left(\frac{\partial}{\partial x_{i}}\left(\left[\frac{\partial V^{k}}{\partial x_{l}}\right]_{k, l}-V^{j} \Gamma^{j}\right)\right. \\
-\left(\left[\frac{\partial V^{k}}{\partial x_{l}}\right]_{k, l}-V^{j} \Gamma^{j}\right) \widehat{\boldsymbol{\Gamma}}_{i} \\
\left.-\widehat{\boldsymbol{\Gamma}}_{i}^{T}\left(\left[\frac{\partial V^{k}}{\partial x_{l}}\right]_{k, l}-V^{j} \boldsymbol{\Gamma}^{j}\right)\right)\left(\nabla \boldsymbol{\Phi}_{\varepsilon}^{\alpha}\right)^{-1} \mathbf{a}^{i} \\
\widehat{\boldsymbol{\Gamma}}_{i}=\left[\Gamma_{i k}^{j}\right]_{j, k} \cdot
\end{gathered}
$$

Here $S=s \circ \boldsymbol{\Phi}_{\varepsilon}^{\alpha}$ denotes a scalar field, while $\mathbf{V}=\mathbf{v} \circ \boldsymbol{\Phi}_{\varepsilon}^{\alpha}$ stands for a vector field $\left(V^{i}=\mathbf{V} \cdot \mathbf{a}_{i}\right.$ are the corresponding covariant components). It is important to be aware that, in the above formulae, summation is taken over repeated indices (Einstein summation convention).

Rather complicated but straightforward calculations based on (34)-(36) provide us with the transformed equations written in the curvilinear coordinates. We write only the terms of order 1 and $\varepsilon$; that is, we neglect the terms with higher powers of $\varepsilon$. For notational simplicity, we omit the nonlinear terms in the momentum equations since those terms will not contribute to the macroscopic model. The equations read

$$
\begin{array}{r}
-\left[\Delta V_{\varepsilon}^{1}+\kappa\left(\cos \alpha\left(\frac{\partial V_{\varepsilon}^{1}}{\partial x_{2}}-\frac{\partial V_{\varepsilon}^{2}}{\partial x_{1}}\right)-\frac{\partial V_{\varepsilon}^{2}}{\partial x_{1}}\right.\right. \\
\left.+\sin \alpha\left(\frac{\partial V_{\varepsilon}^{3}}{\partial x_{1}}-\frac{\partial V_{\varepsilon}^{1}}{\partial x_{3}}\right)\right)
\end{array}
$$

$$
\begin{aligned}
& +\left(\kappa^{2}-2 \kappa\right) V_{\varepsilon}^{1}-\kappa^{\prime} V_{\varepsilon}^{2}+\kappa\left(e_{\alpha} \cdot \tilde{x}\right) \\
& \cdot\left(3 \frac{\partial^{2} V_{\varepsilon}^{1}}{\partial x_{1}^{2}}+\Delta_{x^{\prime}} V_{\varepsilon}^{1}-\kappa \cos \alpha \frac{\partial V_{\varepsilon}^{1}}{\partial x_{2}}\right. \\
& +\kappa \sin \alpha\left(\frac{\partial V_{\varepsilon}^{1}}{\partial x_{3}}+3 \frac{\partial V_{\varepsilon}^{3}}{\partial x_{1}}\right)-3 \kappa^{\prime} V_{\varepsilon}^{2} \\
& \left.\left.-3 \kappa(1+\cos \alpha) \frac{\partial V_{\varepsilon}^{2}}{\partial x_{1}}+\left(\kappa^{2}-6 \kappa\right) V_{\varepsilon}^{1}\right)\right] \\
& +\left(1+\kappa\left(e_{\alpha} \cdot \tilde{x}\right)\right) \frac{\partial Q_{\varepsilon}}{\partial x_{1}}=0
\end{aligned}
$$

$$
\begin{gathered}
-\left[\cos \alpha \Delta V_{\varepsilon}^{2}-\sin \alpha \Delta V_{\varepsilon}^{3}+2 \kappa \frac{\partial V_{\varepsilon}^{1}}{\partial x_{1}}-\kappa \cos ^{2} \alpha \frac{\partial V_{\varepsilon}^{2}}{\partial x_{2}}\right. \\
-\kappa \sin ^{2} \alpha \frac{\partial V_{\varepsilon}^{3}}{\partial x_{3}}+\kappa^{\prime} V_{\varepsilon}^{1}+\frac{\kappa}{2} \sin 2 \alpha\left(\frac{\partial V_{\varepsilon}^{2}}{\partial x_{3}}+\frac{\partial V_{\varepsilon}^{3}}{\partial x_{2}}\right) \\
-\kappa^{2} V_{\varepsilon}^{2}+2 \kappa\left(e_{\alpha} \cdot \tilde{x}\right) \\
\left(\cos \alpha \frac{\partial^{2} V_{\varepsilon}^{2}}{\partial x_{1}^{2}}-\sin \alpha \frac{\partial^{2} V_{\varepsilon}^{3}}{\partial x_{1}^{2}}\right. \\
+2 \kappa \frac{\partial V_{\varepsilon}^{1}}{\partial x_{1}}-\kappa \cos ^{2} \alpha \frac{\partial V_{\varepsilon}^{2}}{\partial x_{2}}-\kappa \sin ^{2} \alpha \frac{\partial V_{\varepsilon}^{3}}{\partial x_{3}} \\
\left.\left.+\frac{\kappa}{2} \sin 2 \alpha\left(\frac{\partial V_{\varepsilon}^{2}}{\partial x_{3}}+\frac{\partial V_{\varepsilon}^{3}}{\partial x_{2}}\right)+\kappa^{\prime} V_{\varepsilon}^{1}-\kappa^{2} V_{\varepsilon}^{2}\right)\right] \\
+\cos \alpha \frac{\partial Q_{\varepsilon}}{\partial x_{2}}-\sin \alpha \frac{\partial Q_{\varepsilon}}{\partial x_{3}}=0,
\end{gathered}
$$

$$
\begin{array}{r}
-\left[\sin \alpha \Delta V_{\varepsilon}^{2}+\cos \alpha \Delta V_{\varepsilon}^{3}-\kappa \cos ^{2} \alpha \frac{\partial V_{\varepsilon}^{3}}{\partial x_{2}}+\kappa \sin ^{2} \alpha \frac{\partial V_{\varepsilon}^{2}}{\partial x_{3}}\right. \\
+\frac{\kappa}{2} \sin 2 \alpha\left(\frac{\partial V_{\varepsilon}^{3}}{\partial x_{3}}-\frac{\partial V_{\varepsilon}^{2}}{\partial x_{2}}\right)+\kappa \tau V_{\varepsilon}^{1}+2 \kappa\left(e_{\alpha} \cdot \tilde{x}\right) \\
\cdot\left(\sin \alpha \frac{\partial^{2} V_{\varepsilon}^{2}}{\partial x_{1}^{2}}+\cos \alpha \frac{\partial^{2} V_{\varepsilon}^{3}}{\partial x_{1}^{2}}-\kappa \cos ^{2} \alpha \frac{\partial V_{\varepsilon}^{3}}{\partial x_{2}}\right. \\
+\kappa \sin ^{2} \alpha \frac{\partial V_{\varepsilon}^{2}}{\partial x_{3}} \\
\left.\left.+\frac{\kappa}{2} \sin 2 \alpha\left(\frac{\partial V_{\varepsilon}^{3}}{\partial x_{3}}-\frac{\partial V_{\varepsilon}^{2}}{\partial x_{2}}\right)+\kappa \tau V_{\varepsilon}^{1}\right)\right] \\
+\sin \alpha \frac{\partial Q_{\varepsilon}}{\partial x_{2}}-\cos \alpha \frac{\partial Q_{\varepsilon}}{\partial x_{3}}=0
\end{array}
$$




$$
\begin{aligned}
\frac{\partial V_{\varepsilon}^{1}}{\partial x_{1}} & -\kappa V_{\varepsilon}^{2}+\frac{\partial V_{\varepsilon}^{2}}{\partial x_{2}}+\frac{\partial V_{\varepsilon}^{3}}{\partial x_{3}} \\
& +2 \kappa\left(e_{\alpha} \cdot \tilde{x}\right)\left(\frac{\partial V_{\varepsilon}^{1}}{\partial x_{1}}-\kappa V_{\varepsilon}^{2}\right)=0
\end{aligned}
$$

with

$$
\mathbf{U}_{\varepsilon}=\mathbf{u}_{\varepsilon} \circ \Phi_{\varepsilon}^{\alpha}=V_{\varepsilon}^{1} \mathbf{t}+V_{\varepsilon}^{2} \mathbf{n}+V_{\varepsilon}^{3} \mathbf{b}, \quad Q_{\varepsilon}=q_{\varepsilon} \circ \Phi_{\varepsilon}^{\alpha} .
$$

3.3. Asymptotic Solution of the Transformed Problem. Now we apply the two-scale asymptotic technique on the transformed problem (37) $-(40)$ posed in $T_{\varepsilon}=(0, \ell) \times \varepsilon B$. More precisely, we expand the unknowns $\mathbf{U}_{\varepsilon}$ and $Q_{\varepsilon}$ in powers of small parameter $\varepsilon$ as follows:

$$
\begin{gathered}
V_{\varepsilon}^{i}(x)=\varepsilon^{2} V_{0}^{i}\left(x_{1}, \frac{x_{2}}{\varepsilon}, \frac{x_{3}}{\varepsilon}\right)+\varepsilon^{3} V_{1}^{i}\left(x_{1}, \frac{x_{2}}{\varepsilon}, \frac{x_{3}}{\varepsilon}\right)+\cdots, \\
i=1,2,3, \\
Q_{\varepsilon}(x)=Q_{0}\left(x_{1}, \frac{x_{2}}{\varepsilon}, \frac{x_{3}}{\varepsilon}\right)+\varepsilon Q_{1}\left(x_{1}, \frac{x_{2}}{\varepsilon}, \frac{x_{3}}{\varepsilon}\right)+\cdots .
\end{gathered}
$$

Plugging the above expansions in (37)-(40), after collecting the terms with equal powers of $\varepsilon$ we are going to obtain the recursive sequence of problems now posed in the $\varepsilon$-independent domain $T=(0, \ell) \times B$. In view of that, we introduce the rescaled variable $y=\left(y_{2}, y_{3}\right)=\left(x_{2} / \varepsilon, x_{3} / \varepsilon\right)$ and use the following notation for partial differential operators:

$$
\begin{gathered}
\nabla_{y}=\frac{\partial}{\partial y_{2}} \mathbf{j}+\frac{\partial}{\partial y_{3}} \mathbf{k}, \\
\Delta_{y} \mathbf{V}=\frac{\partial^{2} \mathbf{V}}{\partial y_{2}^{2}}+\frac{\partial^{2} \mathbf{V}}{\partial y_{3}^{2}}, \\
\operatorname{div}_{y} \mathbf{V}=\frac{\partial V^{2}}{\partial y_{2}}+\frac{\partial V^{3}}{\partial y_{3}}, \\
\mathbf{V}=V^{1} \mathbf{i}+V^{2} \mathbf{j}+V^{3} \mathbf{k} .
\end{gathered}
$$

First, let us note that substituting (42) in momentum equations (38)-(39) implies that $\nabla_{y} Q_{0}=0$; that is, the lowestorder approximation for the transformed pressure depends only on the variable $x_{1}$ going along the pipe. That was to be expected due to the small pipe's thickness. Next, we deduce the problem satisfied by the zero-order approximation for the velocity:

$$
\begin{gathered}
1:-\Delta_{y} \mathbf{V}_{0}+\left(\frac{d Q_{0}}{d x_{1}}, \frac{\partial Q_{1}}{\partial y_{2}}, \frac{\partial Q_{1}}{\partial y_{3}}\right)=0 \quad \text { in } T, \\
\varepsilon: \operatorname{div}_{y} \mathbf{V}_{0}=0 \quad \text { in } T, \\
\varepsilon^{2}: \frac{\partial V_{0}^{1}}{\partial x_{1}}+\operatorname{div}_{y} \mathbf{V}_{1}=0 \quad \text { in } T, \\
\mathbf{V}_{0}=0 \quad \text { on } \Gamma=(0, \ell) \times \partial B, \\
Q_{0}(i)=\frac{1}{\beta \mu_{0}}\left(e^{-\beta q_{0}}-e^{-\beta p_{i}}\right) \quad i=0, \ell .
\end{gathered}
$$

Observe that, at this stage, the angle $\alpha$ is eliminated from the system by adding/substracting (38) and (39) multiplied by $\cos \alpha$ and $\sin \alpha$. Consequently, the system (44) will yield the solution in the following form:

$$
\begin{gathered}
V_{0}^{1}(y)=\frac{1}{4 \beta \mu_{0} \ell}\left(e^{-\beta p_{0}}-e^{-\beta p_{\ell}}\right)\left(|y|^{2}-1\right), \\
Q_{0}\left(x_{1}\right)=\frac{1}{\beta \mu_{0}}\left(e^{-\beta q_{0}}-e^{-\beta p_{0}}\right)+\frac{e^{-\beta p_{0}}-e^{-\beta p_{\ell}}}{\beta \mu_{0} \ell} x_{1}, \\
V_{0}^{2}=V_{0}^{3}=0, \quad Q_{1}=Q_{1}\left(x_{1}\right) .
\end{gathered}
$$

Seeking for the effects of the pipe's distortion, we continue the computation and try to construct the velocity corrector. The $\mathcal{O}(\varepsilon)$ term from (37) provides the following equation for the first component $V_{1}^{1}$ :

$$
\begin{aligned}
\varepsilon:- & \left(\Delta_{y} V_{1}^{1}+\kappa \cos \alpha \frac{\partial V_{0}^{1}}{\partial y_{2}}-\kappa \sin \alpha \frac{\partial V_{0}^{1}}{\partial y_{3}}+\kappa\left(e_{\alpha} \cdot y\right) \Delta_{y} V_{0}^{1}\right) \\
& +\kappa\left(e_{\alpha} \cdot y\right) \frac{d Q_{0}}{d x_{1}}+\frac{d Q_{1}}{d x_{1}}=0 .
\end{aligned}
$$

Taking into account (45), we arrive at

$$
\begin{aligned}
&-\Delta_{y} V_{1}^{1}= \frac{\kappa}{2 \beta \mu_{0} \ell}\left(e^{-\beta p_{0}}-e^{-\beta p_{\ell}}\right)\left(\cos \alpha y_{2}-\sin \alpha y_{3}\right) \\
&-\frac{d Q_{1}}{d x_{1}}\left(x_{1}\right) \quad \text { in } T, \\
& V_{1}^{1}=0 \text { on } \Gamma .
\end{aligned}
$$

Since $Q_{1}=0$ for $x_{1}=0, \ell$, the above system can be solved by putting $Q_{1}=0$ and

$$
\begin{aligned}
V_{1}^{1}\left(x_{1}, y\right)= & \frac{\kappa\left(x_{1}\right)}{16 \beta \mu_{0} \ell}\left(e^{-\beta p_{0}}-e^{-\beta p_{\ell}}\right) \\
& \cdot\left(1-|y|^{2}\right)\left(\cos \alpha y_{2}-\sin \alpha y_{3}\right) .
\end{aligned}
$$

By a simple integration (passing to polar coordinates), one can easily check that $\int_{B} V_{1}^{1}=0$. It means that the pipe's curvature, appearing in the correction for the tangential velocity, affects only the flow profile but not the mean flow.

Finally, from (38)-(40) we deduce the problem for the remaining two components:

$$
\begin{gathered}
\varepsilon:-\Delta_{y} \widetilde{\mathbf{V}}_{1}+\nabla_{y} Q_{2}=0 \quad \text { in } T, \\
\varepsilon^{2}: \operatorname{div}_{y} \mathbf{V}_{1}=0 \quad \text { in } T, \\
\widetilde{\mathbf{V}}_{1}=0 \quad \text { on } \Gamma,
\end{gathered}
$$

where $\widetilde{\mathbf{V}}_{1}=\left(V_{1}^{2}, V_{1}^{3}\right)$. It is well-known that the above system has (unique) trivial solution so we do not observe the desired effects here. 
To conclude this section we write the asymptotic solution of the transformed problem (19)-(22). The velocity part is given by

$$
\begin{aligned}
& \mathbf{u}_{\varepsilon}^{\text {approx }}(z)=\mathscr{V}_{\varepsilon}(x), \quad z=\Phi_{\varepsilon}^{\alpha}(x), \\
\mathscr{V}_{\varepsilon}(x)= & \left(\varepsilon^{2} V_{0}^{1}+\varepsilon^{3} V_{1}^{1}\right) \mathbf{t} \\
= & \frac{e^{-\beta p_{\ell}}-e^{-\beta p_{0}}}{4 \beta \mu_{0} \ell}\left(\varepsilon^{2}-x_{2}^{2}-x_{3}^{2}\right) \\
& \cdot\left(1-\frac{\kappa\left(x_{1}\right)}{4}\left(\cos \alpha x_{2}-\sin \alpha x_{3}\right)\right) \mathbf{t}_{\varepsilon}\left(x_{1}\right) .
\end{aligned}
$$

For the transformed pressure we obtain

$$
\begin{gathered}
q_{\varepsilon}^{\text {approx }}(z)=Q_{\varepsilon}(x), \quad z=\Phi_{\varepsilon}(x) \\
Q_{\varepsilon}(x)=Q_{0}\left(x_{1}\right)=\frac{1}{\beta \mu_{0}}\left(e^{-\beta q_{0}}-e^{-\beta p_{0}}\right)+\frac{e^{-\beta p_{0}}-e^{-\beta p_{\ell}}}{\beta \mu_{0} \ell} x_{1} .
\end{gathered}
$$

3.4. Back to the Original Problem. Now we apply the inverse transformation, that is, we reconstruct the original pressure from (16):

$$
p_{\varepsilon}^{\text {approx }}=\frac{1}{\beta} \ln \left(\frac{1}{e^{-\beta q_{0}}-\beta \mu_{0} q_{\varepsilon}^{\text {approx }}}\right) .
$$

In view of (51), we deduce

$$
\begin{gathered}
p_{\varepsilon}^{\text {approx }}(z)=\mathscr{P}_{\varepsilon}(x), \quad z=\Phi_{\varepsilon}(x), \\
\mathscr{P}_{\varepsilon}(x)=\frac{1}{\beta} \ln \left(\frac{1}{e^{-\beta p_{0}}+\left(\left(e^{-\beta p_{\ell}}-e^{-\beta p_{0}}\right) / \ell\right) x_{1}}\right) .
\end{gathered}
$$

This represents our asymptotic approximation for the effective pressure. Obviously, it is well-defined since $p_{0}>$ $p_{\ell}$ implying $e^{-\alpha p_{\ell}}>e^{-\alpha p_{0}}$. Next, notice that the effective pressure does not depend on $q_{0}$ at all. This means that, throughout the whole process, the parameter $q_{0}$ has been just an auxiliary parameter; that is, it was justified to choose the parameter $q_{0}$ such that (23) holds. If we compare $p_{\varepsilon}^{\text {approx }}$ with the approximation for straight-pipe flow derived in [21], we do not observe any effects coming due to the pipe's distortion. Those effects could be retrieved by computing lower-order terms in the pressure expansion. However, those terms would serve only for the convergence proof; that is, they would not influence the effective flow.

For the velocity approximation, we have

$$
\begin{gathered}
\mathbf{u}_{\varepsilon}^{\text {approx }}(z)=\mathscr{V}_{\varepsilon}(x), \quad z=\Phi_{\varepsilon}^{\alpha}(x), \\
\mathscr{V}_{\varepsilon}(x)=\frac{e^{-\beta p_{\ell}}-e^{-\beta p_{0}}}{4 \beta \mu_{0} \ell}\left(\varepsilon^{2}-x_{2}^{2}-x_{3}^{2}\right) \\
\cdot\left(1-\frac{\kappa\left(x_{1}\right)}{4}\left(e_{\alpha} \cdot \tilde{x}\right)\right) \mathbf{t}_{\varepsilon}\left(x_{1}\right)
\end{gathered}
$$

and we can clearly detect the effects of the pipe's curvedness. Indeed, though its main part remains in the Poiseuille form (in direction tangential to the central line of the pipe-note that the tangent is not constant vector), the flow profile is not perfectly parabolic any more and it is being corrected. More precisely, the tangential corrector is proportional to the curvature of the pipe's central curve, and that should be acknowledged in the precise analysis of the curved-pipes flow.

Finally, to emphasize the importance of the obtained result, let us compare it with the one from [12] derived for Newtonian (i.e., the constant viscosity) case. The differences coming due to the effects of the pressure-dependent viscosity are particularly observed in the approximation for the pressure (53). Instead of simple linear function $Q_{\mathcal{E}}\left(x_{1}\right)=$ $\left(\left(p_{\ell}-p_{0}\right) / \ell\right) x_{1}+p_{0}$, here we obtain a new asymptotic solution clearly acknowledging the viscosity-pressure dependence of the Barus law. The velocity approximation (54) also feels the effects of the variable viscosity. Indeed, the constant term multiplying the approximation is consistent with the viscosity obeying the Barus law and that was to be expected. The main difference with respect to the Newtonian case is that the velocity is exclusively in the tangential direction; that is, no effects of the central curve's flexion and torsion appear in the direction of the normal or binormal.

\section{Concluding Remarks}

In the previous section, we formally derived an asymptotic model describing the flow of incompressible fluid with a pressure-dependent viscosity through a thin (or long) curved pipe. We assumed that the pressure-dependent viscosity obeyed Barus formula (most commonly used in the engineering community) and worked with physically relevant Dirichlet boundary conditions. Since the pipe's central curve is assumed to be a general (generic) curve, the applicability of the obtained result is broad. Moreover, by obtaining the explicit expressions (53)-(54) for the pressure and velocity distribution, we believe that the result is very relevant with regard to numerical simulations. The effects of pipe's distortion are clearly detected and the difference between classical Newtonian and non-Newtonian flow is established as well.

From the rigorous mathematical point of view, we should link our formally obtained solution with the original solution by proving some kind of convergence result. It can be accomplished by evaluating the difference between those two solutions in the appropriate rescaled norm. Though rigorous justification is out of scope of the present paper, let us comment on this as well. Since $\operatorname{det} \nabla \Phi_{\varepsilon}^{\alpha}=\mathscr{O}(1)$, it is easy to confirm that the appropriate norm is, in fact, $\|\cdot\|_{\varepsilon}=\varepsilon^{-1}\|\cdot\|_{L^{2}\left(\Omega_{\varepsilon}^{\alpha}\right)}$. Thus, the idea is to derive the error estimates (expressed in this norm) for the asymptotic solution of the transformed problem and then to use the continuity of the inverse transformation. The first part can be done using techniques from $[10,12]$ developed for curved geometries (see also $[8,9])$, while the second part is straightforward.

To conclude, it is important to emphasize that the presented approach can be extended to a case of general viscosity-pressure relation $p \mapsto \mu(p)$ satisfied by Barus law and other empiric laws. To assure the well-posedness (see [25]), we only have to impose some technical conditions 
addressing the behavior of the viscosity for large negative pressures being, obviously, irrelevant from the physical point of view. Instead of (16), we simply introduce $q_{\varepsilon}$ as $B\left(p_{\varepsilon}\right)=$ $\int_{q_{0}}^{p_{\varepsilon}}(d \xi / \mu(\xi))$ and apply the same procedure as presented above. We refer the reader to [21] for details.

\section{Conflict of Interests}

The author declares that there is no conflict of interests regarding the publication of this paper.

\section{Acknowledgments}

The author of this work has been supported by the Croatian Science Foundation (Scientific Project no. 3955: Mathematical Modeling and Numerical Simulations of Processes in Thin or Porous Domains). The author would like to thank the referee for valuable comments and suggestions that helped to improve the paper.

\section{References}

[1] G. G. Stokes, "On the theories of the internal friction of fluids in motion, and of the equilibrium and motion of elastic solids," Transactions of the Cambridge Philosophical Society, vol. 8, pp. 287-305, 1845.

[2] A. Goubert, J. Vermant, P. Moldenaers, A. Göttfert, and B. Ernst, "Comparison of measurement techniques for evaluating the pressure dependence of the viscosity," Applied Rheology, vol. 11, no. 1, pp. 26-37, 2001.

[3] Z. Silber-Li, H. Cui, Y. Tan, and P. Tabeling, "Flow characteristics of liquid with pressure-dependent viscosities in microtubes," Acta Mechanica Sinica, vol. 22, no. 1, pp. 17-21, 2006.

[4] C. Schoof, "Pressure-dependent viscosity and interfacial instability in coupled ice-sediment flow," Journal of Fluid Mechanics, vol. 570, pp. 227-252, 2007.

[5] H. E. Park, S. T. Lim, H. M. Laun, and J. M. Dealy, "Measurement of pressure coefficient of melt viscosity: drag flow versus capillary flow," Rheologica Acta, vol. 47, no. 9, pp. 1023-1038, 2008.

[6] C. Schaschke, I. Fletcher, and N. Glen, "Density and viscosity measurement of diesel fuels at combined high pressure and elevated temperature," Processes, vol. 1, no. 2, pp. 30-48, 2013.

[7] C. Barus, "Isothermals, isopiestics and isometrics relative to viscosity," The American Journal of Science, vol. 45, no. 266, pp. 87-96, 1893.

[8] E. Marušić-Paloka and I. Pažanin, "Fluid flow through a helical pipe," Zeitschrift für Angewandte Mathematik und Physik, vol. 58, no. 1, pp. 81-99, 2007.

[9] E. Marušić-Paloka and I. Pažanin, "On the effects of curved geometry on heat conduction through a distorted pipe," Nonlinear Analysis. Real World Applications, vol. 11, no. 6, pp. 45544564, 2010.

[10] I. Pažanin, "Asymptotic behavior of micropolar fluid flow through a curved pipe," Acta Applicandae Mathematicae, vol. 116, no. 1, pp. 1-25, 2011.

[11] C. Y. Wang, "On the low-Reynolds-number flow in a helical pipe," Journal of Fluid Mechanics, vol. 108, pp. 185-194, 1981.
[12] E. Marušić-Paloka, "The effects of flexion and torsion for a fluid flow through a curved pipe," Applied Mathematics and Optimization, vol. 44, no. 3, pp. 245-272, 2001.

[13] J.-W. Wang and J. R. G. Andrews, "Numerical simulation of flow in helical ducts," AIChE Journal, vol. 41, no. 5, pp. 1071-1080, 1995.

[14] E. Marušić-Paloka and I. Pažanin, "On reactive solute transport through a curved pipe," Applied Mathematics Letters, vol. 24, no. 6, pp. 878-882, 2011.

[15] I. Pažanin, "Investigation of micropolar fluid flow in a helical pipe via asymptotic analysis," Communications in Nonlinear Science and Numerical Simulation, vol. 18, no. 3, pp. 528-540, 2013.

[16] M. Renardy, "Parallel shear flows of fluids with a pressuredependent viscosity," Journal of Non-Newtonian Fluid Mechanics, vol. 114, no. 2-3, pp. 229-236, 2003.

[17] M. Vasudevaiah and K. R. Rajagopal, "On fully developed flows of fluids with a pressure dependent viscosity in a pipe," Applications of Mathematics, vol. 50, no. 4, pp. 341-353, 2005.

[18] A. Kalogirou, S. Poyiadji, and G. C. Georgiou, "Incompressible Poiseuille flows of Newtonian liquids with a pressuredependent viscosity," Journal of Non-Newtonian Fluid Mechanics, vol. 166, no. 7-8, pp. 413-419, 2011.

[19] K. D. Housiadas, G. C. Georgiou, and R. I. Tanner, "A note on the unbounded creeping flow past a sphere for Newtonian fluids with pressure-dependent viscosity," International Journal of Engineering Science, vol. 86, pp. 1-9, 2015.

[20] S. Poyiadji, K. D. Housiadas, K. Kaouri, and G. C. Georgiou, "Asymptotic solutions of weakly compressible Newtonian Poiseuille flows with pressure-dependent viscosity," European Journal of Mechanics. B. Fluids, vol. 49, pp. 217-225, 2015.

[21] E. Marušić-Paloka and I. Pažanin, "A note on the pipe flow with a pressure-dependent viscosity," Journal of Non-Newtonian Fluid Mechanics, vol. 197, pp. 5-10, 2013.

[22] I. Pažanin, "On the helical pipe flow with a pressure-dependent viscosity," Theoretical and Applied Mechanics Letters, vol. 4, no. 6, Article ID 062006, 2014.

[23] M. Germano, "On the effect of torsion on a helical pipe flow," Journal of Fluid Mechanics, vol. 125, pp. 1-8, 1982.

[24] M. Germano, "The Dean equations extended to a helical pipe flow," Journal of Fluid Mechanics, vol. 203, pp. 289-305, 1989.

[25] E. Marušić-Paloka, "An analysis of the Stokes system with pressure dependent viscosity," Rendiconti dell'Istituto di Matematica dell'Università di Trieste, vol. 46, pp. 1-14, 2014. 


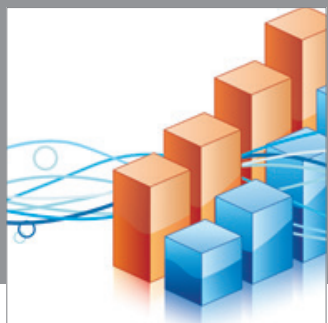

Advances in

Operations Research

mansans

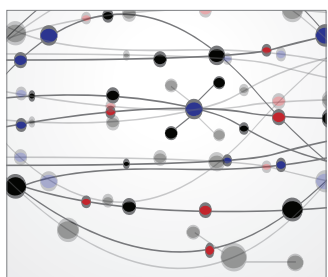

The Scientific World Journal
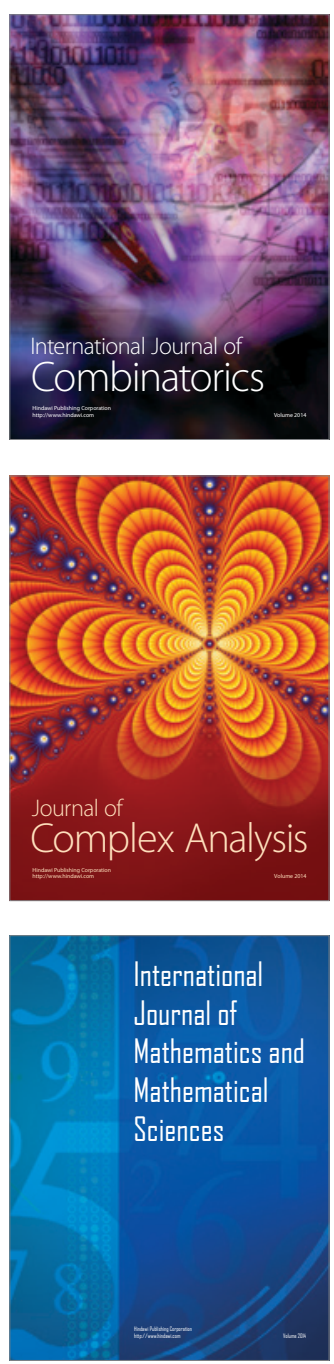
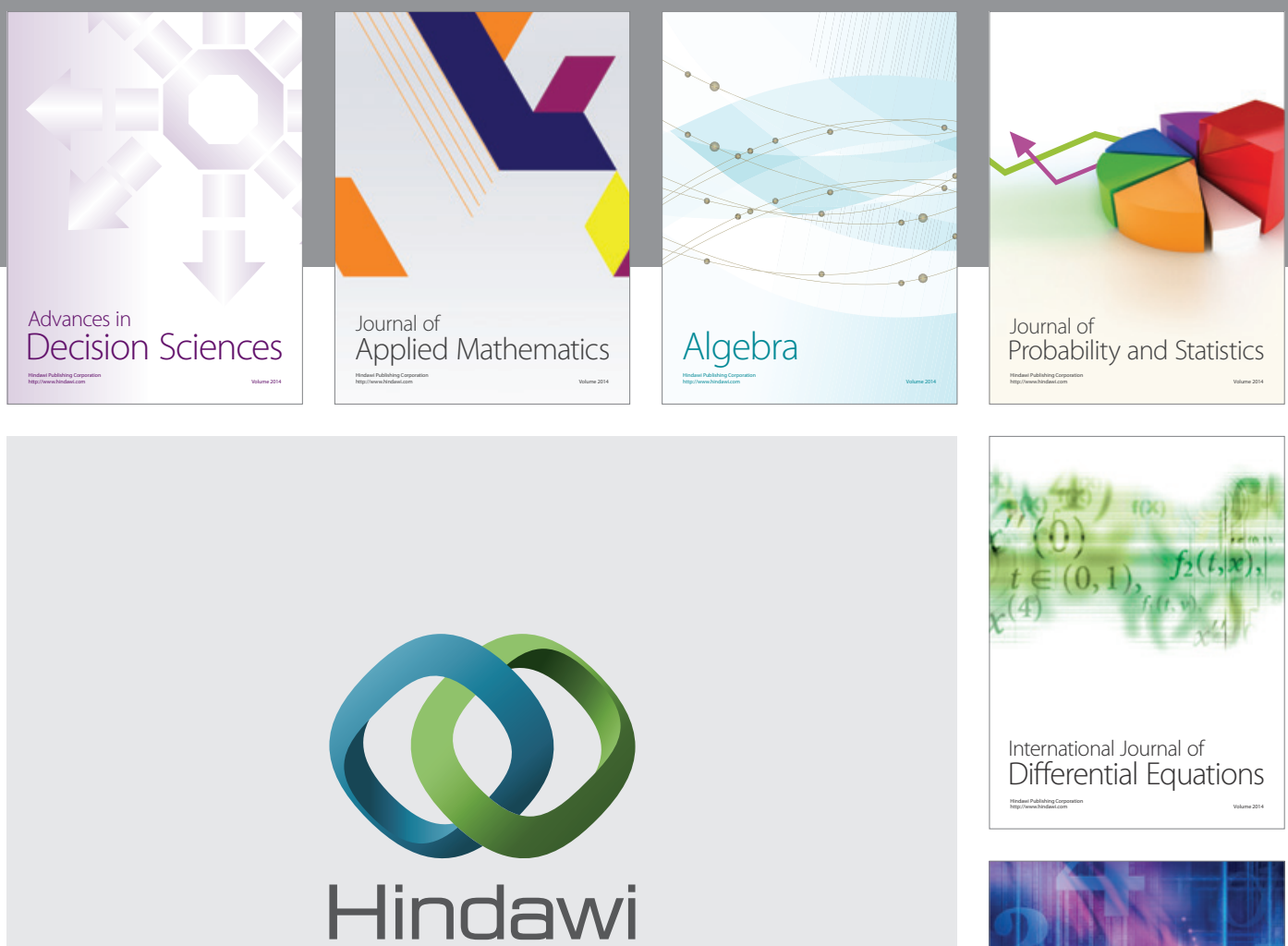

Submit your manuscripts at http://www.hindawi.com
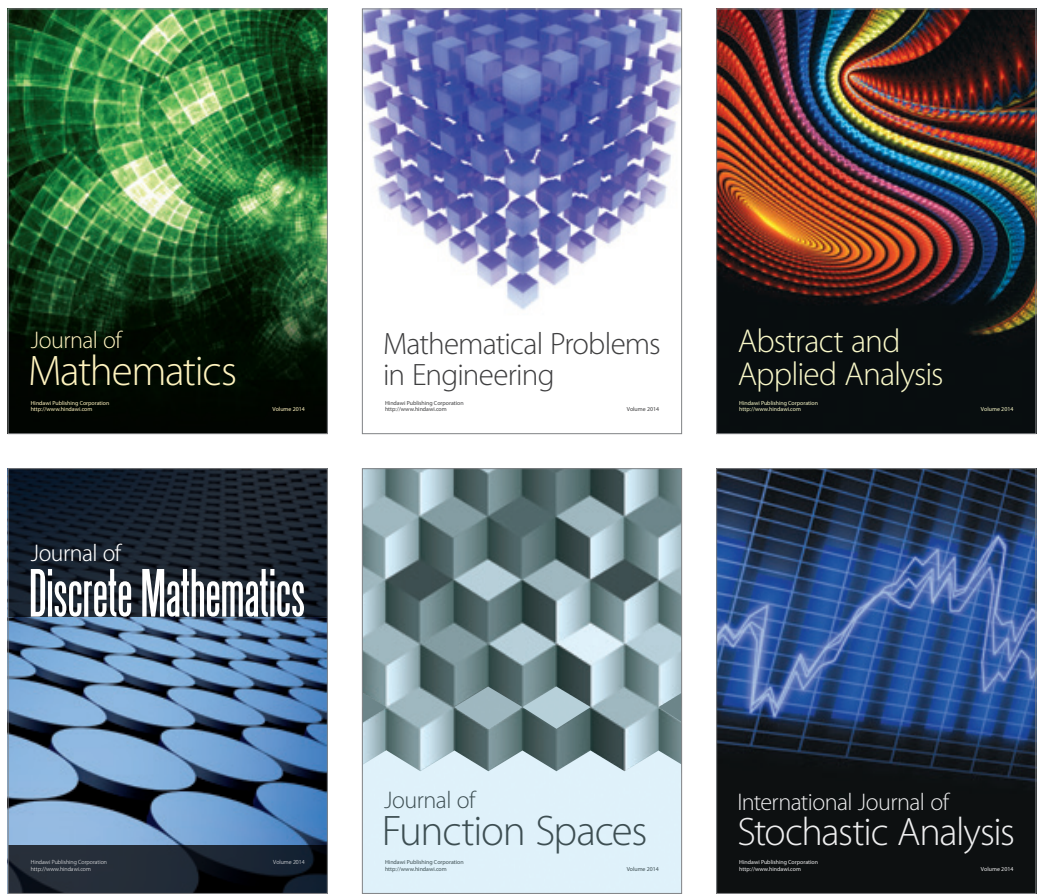

Journal of

Function Spaces

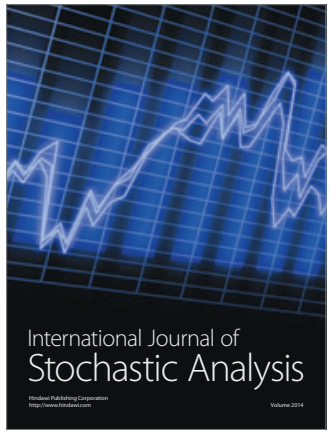

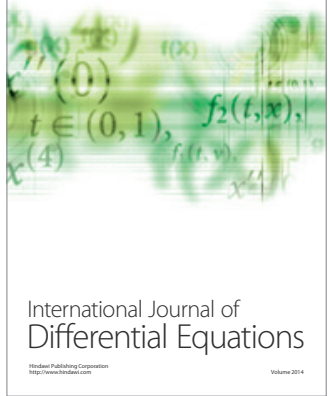
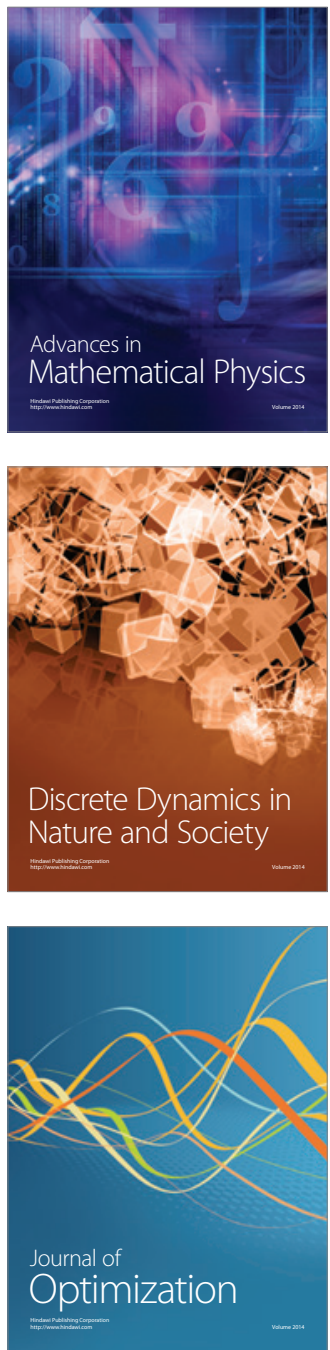